\title{
Oesophagectomy rates and post-resection outcomes in patients with cancer of the oesophagus and gastro-oesophageal junction: a population-based study using linked health administrative linked data
}

\author{
Efty P Stavrou ${ }^{1,3^{*}}$, Robyn Ward ${ }^{1}$ and Sallie-Anne Pearson ${ }^{1,2}$
}

\begin{abstract}
Background: Hospital performance is being benchmarked increasingly against surgical indicators such as 30-day mortality, length-of-stay, survival and post-surgery complication rates. The aim of this paper was to examine oesophagectomy rates and post-surgical outcomes in cancers of the oesophagus and gastro-oesophageal junction and to determine how the addition of gastro-oesophageal cancer to oesophageal cancer impacts on these outcomes.

Methods: Our study population consisted of patients with a primary invasive oesophageal or gastro-oesophageal cancer identified from the NSW Cancer Registry from July 2000-Dec 2007. Their records were linked to the hospital separation data for determination of resection rates and post-resection outcomes. We used multivariate logistic regression analyses to examine factors associated with oesophagectomy and post-resection outcomes.

Cox-proportional hazard regression analysis was used to examine one-year cancer survival following oesophagectomy.

Results: We observed some changes in resection rates and surgical outcomes with the addition of gastro-oesophageal cancer patients to the oesophageal cancer cohort. 14.6\% of oesophageal cancer patients and $26.4 \%$ of gastro-oesophageal cancer patients had an oesophagectomy; an overall oesophagectomy rate of $18.2 \%$ in the combined cohort. In the combined cohort, oesophagectomy was associated with younger age, being male and Australian-born, having non-metastatic disease or adenocarcinoma and being admitted in a co-located hospital. Rates of length-of-stay $>28$ days (20.9\% vs 19.7\%), 30-day mortality (3.8\% vs $2.7 \%$ ) and one-year survival post-surgery (24.5\% vs $23.1 \%$ ) were similar between oesophageal cancer alone and the combined cohort; whilst 30-day complication rates were $21.5 \%$ versus $17.0 \%$ respectively. Some factors statistically associated with post-resection complication in oesophageal cancer alone were not significant in the overall cohort. Poorer post-resection outcomes were associated with some patient (older age, birthplace) and hospital-related characteristics (fiscal sector, area health service).

(Continued on next page)
\end{abstract}

\footnotetext{
* Correspondence: eftyhia.stavrou@sydney.edu.au

'Lowy Cancer Research Centre, Prince of Wales Clinical School, University of

New South Wales, Sydney, NSW, Australia

${ }^{3}$ Kolling Institute of Medical Research, University of Sydney, St Leonards, NSW

2065, Australia

Full list of author information is available at the end of the article
} 
(Continued from previous page)

Conclusion: Outcomes following oesophagectomy in oesophageal and gastro-oesophageal cancer patients in NSW are within world benchmarks. Our study demonstrates that the inclusion of gastro-oesophageal cancer did alter some outcomes compared to analysis based solely on oesophageal cancer. As such, care must be taken with analyses based on administrative health data to capture all populations eligible for treatment and to understand the contribution of these subpopulations to overall outcomes.

Keywords: Hospital outcomes, Oesophageal cancer, Administrative health data, Data linkage

\section{Background}

Accountability and transparency in health care, at individual and institutional levels, has gained increasing importance in recent years [1-3]. This growing focus on quality of practise and safety of services has implications for patients, clinicians, administrators and policy makers. Quality assessments of hospital performance are now conducted routinely throughout the world, the results of which are made available to all stakeholders. Information of this kind has been used for the purposes of benchmarking, so as to improve performance across all institutions [4] and to empower the general public to make informed decisions about where they choose to receive care.

Surgical resection for curable oesophageal and gastrooesophageal cancer is the mainstay of treatment for all patients who are fit for major surgery. However, despite being the only curative treatment for oesophageal/ gastro-oesophageal cancer patients, oesophagectomy is associated with significant operative morbidity and mortality [5-7]. Key performance measures of oesophagectomy include 30-day mortality, hospital length-of-stay and post-surgery complication rate [8-17]. Factors influencing outcomes following oesophagectomy include hospital/ surgeon factors (peer group/volume, surgical experience), tumour stage, histology and location, surgery type and patient comorbidity; with better outcomes reported in high volume hospitals, patients with non-metastatic disease (approximately $60 \%$ of patients), adenocarcinoma (the incidence of which is increasing), and when transhiatal oesophagectomy is performed [18-25].

In Australia, there has been keen interest in using administrative datasets to examine hospital performance [26-28] with surgeons, administrators and patients having endorsed independent review of surgical care [29]. We previously examined patient outcomes for oesophageal cancer following oesophagectomy in one Australian state using data linkage of administrative datasets [9]. We found oesophagectomy was performed with satisfactory outcomes however resection rates were lower than reported elsewhere, as other studies included cancer of the gastro-oesophageal junction in their analyses since gastro-oesophageal cancer is treated in a similar manner to oesophageal cancer [30,31].
Therefore, our aim was to examine rates of oesophogectomy and post-resection outcomes for patients diagnosed with oesophageal cancer or gastro-oesophageal cancer in the state of New South Wales (NSW) between 2000 and 2007. Specifically, we examined the way in which the inclusion of gastro-oesophageal cancer to the oesophageal cancer cohort impacted on resection rates and post-resection outcomes.

\section{Methods}

\section{Setting}

Australia has a publicly-funded universal health care system and health care data is collected by Commonwealth and state-based jurisdictions. The Australian Government Department of Human Services administer the Medicare Benefits Scheme (MBS; which provides free or subsidised treatment by health professionals and fully subsidised treatment in public hospitals) and the Pharmaceutical Benefits Scheme (PBS; which provides subsidised access to prescription drugs). The individual state and territory health authorities are responsible for the collection of public and private hospital separation data within their jurisdiction. However, crossjurisdictional data sets have not been linked routinely due to legislative and privacy issues.

Our current study uses routinely collected hospital separation data from New South Wales (NSW). NSW is the largest jurisdiction in Australia with eight area health districts (at the time this research commenced) and consists of more than 200 public and 80 private hospitals, some of which are in proximity to each other (colocated). As such medical specialists practise at both the public and affiliated private hospital.

\section{Study population}

Our study population consisted of patients with a primary invasive oesophageal and gastro-oesophageal cancer (International Classification of Diseases v3 [ICD-O-3] codes C15.0-C15.9 and C16.0).

\section{Data sources and linkage}

We accessed and linked two population-based data sets in this study: 
NSW Central Cancer Registry (1 July 2000-31Dec2007) (CCR) receives notifications of cancer in NSW and maintains a record of all cancer cases diagnosed in NSW residents since 1972. The registry is managed according to the International Association of Cancer Registries (IACR) rules [32] and is one of the few Australian Cancer Registries to record degree-of-spread at first diagnosis for all solid malignant tumours [33]. Degree-of-spread is assigned by the NSW CCR into one of four summary stages (localised, regional, distant or unknown) [25]. Histology type was based on Berg groupings of ICD-O-3 classifications and grouped as adenocarcinomas, squamous cell carcinoma (SCC) or other.

Admitted Patient Data Collection (1 July 2000 - 30 June 2008) - is a census of all inpatient separations from all public, private and repatriation hospitals, private day procedures centres and public nursing homes in NSW. We identified surgical resections in the separations using the International Classification of Diseases (ICD_10_AM) procedural block codes for oesophagectomy by abdominal and thoracic/cervical/transthoracic mobilisation and endoscopic mucosal resection 0858-0860 (if recorded) [34].

Data linkage was undertaken by a third-party, the NSW Centre for Health Record Linkage (CHeReL) using best practice privacy preserving protocols. Using probabilistic linkage, $\mathrm{CHeReL}$ identified all appropriate records from the APDC and CCR. They then assigned unique identifiers (or Project Person Number: PPN) to the APDC and CCR files. We received two individual data files and linked the unit-record data using the unique PPNs.

\section{Outcomes measures and statistical analyses}

We reported oesophagectomy rates for oesophageal cancer, gastro-oesophageal cancer and the combined cohort. As oesophagectomy is most beneficial for, and is associated with, non-metastatic disease and patients with adenocarcinoma, we also examined rates of surgical resection and factors associated with surgical resection and post-surgical outcomes for this sub-group of patients. Multivariate logistic regression was used to determine which factors were associated with (i) receiving an oesophagectomy and (ii) the post-resection outcomes of: longer length of hospital stay ( $>28$ days); 30-day complication and 30-day mortality. Post resection complications arising within 30 days were derived from ICD-10-AM diagnostic codes and included any post-procedural disorders or complications, haemorrhages, pulmonary and cardiac complications.

Cox proportional hazard regression survival analysis was also undertaken to examine one-year survival following oesophagectomy. We followed patients diagnosed with oesophageal cancer or cancer of the gastro-oesophageal junction in the NSW CCR to $31^{\text {st }}$ December, 2007 for death from the cancer.

We did not identify individual hospitals in this study, rather we grouped hospitals by health service (identified as Area 1 - Area 8) and according to fiscal sector (public co-located; private co-located; public non co-located; private non co-located). We used the Charlson Comorbidity Index (CCI) to account for comorbidity before cancer diagnosis [35]. This index is commonly used to adjust for comorbidity and was derived from ICD-10AM diagnostic codes as recorded in any hospital separation prior to and including the date of cancer diagnosis $[36,37]$. Other covariates of interest included patient factors of age at diagnosis, gender, Australian or overseas born, tumour location and surgical procedure (where appropriate).

Statistical significance was taken at the $\mathrm{p}<0.05$ level. All statistical analysis was performed using SAS version 11 .

\section{Ethical approval}

This research was approved by the NSW Population and Health Service Research Ethics Committee (2010/05/235).

\section{Results}

\section{Cohort Characteristics (Table 1)}

Our cohort comprised 5,024 patients, 3,456 (69\%) of whom were diagnosed with oesophageal cancer and 1,568 with gastro-oesophageal cancer.

More than half of oesophageal cancer patients were $\geq 70$ years, $67 \%$ were male, $45 \%$ were diagnosed with adenocarcinoma, $20 \%$ with regional spread and $18 \%$ with distant spread-of-disease. The vast majority (90\%) of oesophageal cancer patients were defined by the CCI as having no pre-existing comorbidity. Similarly, most gastro-oesophageal patients were $\geq 70$ years and male. Owing to the nature of gastro-oesophageal cancer, $89 \%$ of this group were diagnosed with adenocarcinoma, 35\% with regional and $25 \%$ with distant disease spread. The addition of gastro-oesophageal patients to the cohort increased the proportion of patients in the overall cohort with adenocarcinoma to approximately $60 \%$, and patients with regional or distant spread increased from $38 \%$ to $45 \%$.

\section{Rates of surgical resection and factors associated with receiving surgical resection (Tables 2,3 )}

The rate of resection in oesophageal cancer patients was $14.6 \%$, whereas $26.4 \%$ of gastro-oesophageal patients underwent surgical resection. The addition of gastrooesophageal cancer patients to the cohort increased the overall surgery rates to $18.2 \%$.

The majority of oesophageal cancer patients who underwent resection were $<70$ years $(71 \%)$, male $(76 \%)$, 
Table 1 Characteristics of patients diagnosed with oesophageal (C15) and gastro-oesophageal cancer (C160)

\begin{tabular}{|c|c|c|c|}
\hline \multirow[t]{3}{*}{ Variable } & C15 & C160 & C15 \& C160 \\
\hline & $\mathrm{N}=3,456$ & $\mathrm{~N}=1,568$ & $\mathrm{~N}=5,024$ \\
\hline & $\%$ & $\%$ & $\%$ \\
\hline
\end{tabular}

Age at diagnosis

group (years)

\begin{tabular}{lllllll}
$<60$ & 657 & 19.0 & 402 & 25.6 & 1,059 & 21.1 \\
$60-69$ & 840 & 24.3 & 399 & 25.4 & 1,239 & 24.7 \\
$70-79$ & 1,048 & 30.3 & 486 & 31.0 & 1,534 & 30.5 \\
$80+$ & 911 & 26.4 & 281 & 17.9 & 1,192 & 23.7 \\
\hline
\end{tabular}

\begin{tabular}{lllllll}
\hline Gender & & & & & & \\
Female & 1,159 & 33.5 & 362 & 23.1 & 1,521 & 30.3 \\
Male & 2,297 & 66.5 & 1,206 & 76.9 & 3,503 & 69.7 \\
\hline Country of Birth & & & & & & \\
Australia & 2,497 & 72.2 & 1,063 & 67.8 & 3,560 & 70.9 \\
Other & 959 & 37.8 & 505 & 32.2 & 1,464 & 29.1 \\
Histology group & & & & & & \\
SCC & 1,478 & 42.8 & 70 & 4.5 & 1,548 & 30.8 \\
Adenocarcinomas & 1,549 & 44.8 & 1,398 & 89.2 & 2,947 & 58.7 \\
Other & 293 & 8.5 & 79 & 5.0 & 372 & 7.4 \\
\hline
\end{tabular}

Degree of Spread

\begin{tabular}{lllllll} 
Localised & 1,290 & 37.3 & 435 & 27.7 & 1,725 & 34.3 \\
Regional & 692 & 20.0 & 543 & 34.6 & 1,235 & 24.6 \\
Distant & 622 & 18.0 & 387 & 24.7 & 1,009 & 20.1 \\
Unknown & 721 & 20.9 & 203 & 12.9 & 924 & 18.4 \\
\hline Tumour location & & & & & & \\
Upper & 297 & 8.6 & & & 297 & 5.9 \\
Middle & 470 & 13.8 & & & 470 & 9.4 \\
Lower & 1,528 & 45.0 & & & 1,528 & 30.9 \\
NOS* $_{\text {GOJ* }}^{1,113}$ & 32.7 & & & 1,113 & 22.2 \\
\hline
\end{tabular}

Charlson comorbidity

$\begin{array}{lllllll}0 & 3,098 & 89.6 & 1,431 & 91.2 & 4,529 & 90.1 \\ 1,2 & 331 & 9.6 & 124 & 7.9 & 455 & 9.1 \\ 3+ & 27 & 0.8 & 13 & 0.8 & 40 & 0.8\end{array}$

${ }^{*} \mathrm{NOS}=$ not otherwise specified; $\mathrm{GOJ}=$ gastro-oesophageal junction.

born in Australia (68\%), had no pre-existing comorbidity $(70 \%)$ and were diagnosed with adenocarcinoma $(62 \%)$ and with loco-regional disease at the time of notification (85\%). Gastro-oesophageal cancer patients had even higher proportions of males $(87 \%)$ and adenocarcinoma patients (93\%) who underwent resection. After adjustment for covariates, surgical resection for oesophageal cancer patients was associated with younger age $(p<0.001)$, adenocarcinoma $(p<0.001)$, non-metastatic disease $(p<0.001)$, no pre-existing comorbidity $(p=0.006)$ and the resection being conducted in a public or private co-located hospital $(p<0.001)$ (Table 2$)$. Not surprisingly the addition of gastro-oesophageal patients to the cohort increased patient numbers and resulted in some additional significant predictors of surgical resection (male gender [aOR=1.31 95\% CI 1.05-1.64], born in Australia $[\mathrm{aOR}=1.32$, 95\% CI 1.09-1.61] and undergoing surgery at specific area health services).

\section{Sub-analyses of patients with non-metastatic adenocarcinoma}

When restricted to the 2,155 non-metastatic adenocarcinoma patients, the addition of gastro-oesophageal cancer patients increased the overall surgery rate to $29.6 \%$ (from $26.1 \%$ for oesophageal cancer alone). Patient and hospital factors associated for surgery in this sub-cohort were the same as the entire cohort. The addition of the gastro-oesophageal patients again added the significant predictors of surgical resection (male gender $[\mathrm{aOR}=2.03$ 95\%CI 1.44-2.85], being born in Australia [aOR=1.41, $95 \%$ CI 1.35-3.44] and undergoing surgery at specific area health services).

\section{Outcomes following surgery (Table 4)}

Rates of hospital length-of-stay $>28$ days $(20.9 \%$ and $19.7 \%), 30$-day mortality (3.8\% and $2.7 \%$ ) and one-year cancer survival $(75.5 \%$ and $76.9 \%)$ were similar for oesophageal cancer and the combined cohort respectively, but differed by $4.5 \%$ for 30 -day complication rates (21.5\% and $17.0 \%)$. Mean one year survival time for the combined cohort was 328 days (SD 48 days).

When investigating the factors associated with longer length of hospital stay and one-year survival, the inclusion of cancer of the gastro-oesophageal junction did not alter the trends established with that of oesophageal cancer alone. However, due to increased precision, some of the associations that were not statistically significant in oesophageal cancer alone became significant in the overall cohort. For longer length of stay these factors were: being Australian born $(\mathrm{aOR}=1.49,95 \% \mathrm{CI} 1.01-2.17)$ and having surgery in a public co-located hospital $(\mathrm{aOR}=2.10$, $95 \%$ CI 1.17-3.78). Other factors associated with length of stay $>28$ days included older age $(p<0.001)$, having an admission in a public hospital $(\mathrm{p}<0.001)$ and not being admitted in certain area health services. Poorer survival in the overall cohort was associated with older age $(p<0.001)$ and private non-co-located hospitals compared with private co-located hospitals $(\mathrm{aHR}=2.20$, 95\%CI 1.03-4.70).

We did not observe any significant associations for 30 day post-resection complication in the overall cohort. However, in the oesophageal cohort we found older age and some area health services were associated with 
Table 2 Patient characteristics, location and surgery type of cohort who received resection

\begin{tabular}{|c|c|c|c|c|c|c|}
\hline \multirow[t]{3}{*}{ Variable } & \multirow{2}{*}{\multicolumn{2}{|c|}{$\frac{\mathrm{C} 15}{\mathrm{~N}=3,456}$}} & \multirow{2}{*}{\multicolumn{2}{|c|}{$\begin{array}{l}\mathrm{C} 160 \\
\mathrm{~N}=1,568\end{array}$}} & \multirow{2}{*}{\multicolumn{2}{|c|}{$\frac{C 15 \& C 160}{N=5,024}$}} \\
\hline & & & & & & \\
\hline & $\mathrm{n}$ & $\%$ & $n$ & $\%$ & $n$ & $\%$ \\
\hline Oesophagectomy Yes & 503 & 14.6 & 412 & 26.3 & 915 & 18.2 \\
\hline \multicolumn{7}{|c|}{ PATIENT CHARACTERISTICS } \\
\hline \multicolumn{7}{|l|}{ Age at diagnosis (years) } \\
\hline$<60$ & 164 & 4.7 & 137 & 8.7 & 301 & 6.0 \\
\hline $60-69$ & 195 & 5.6 & 137 & 8.7 & 332 & 6.6 \\
\hline 70-79 & 116 & 3.4 & 120 & 7.7 & 236 & 4.7 \\
\hline $80+$ & 28 & 0.8 & 18 & 1.1 & 46 & 0.9 \\
\hline \multicolumn{7}{|l|}{ Gender } \\
\hline Female & 118 & 3.4 & 54 & 3.4 & 172 & 3.4 \\
\hline Male & 385 & 11.1 & 358 & 22.8 & 743 & 14.8 \\
\hline \multicolumn{7}{|l|}{ Country of Birth } \\
\hline Australia & 345 & 10.0 & 291 & 18.6 & 636 & 12.7 \\
\hline Other & 158 & 4.6 & 121 & 7.7 & 279 & 5.6 \\
\hline \multicolumn{7}{|l|}{ Histology group } \\
\hline SCC & 169 & 4.9 & 12 & 0.8 & 181 & 3.6 \\
\hline Adenocarcinomas & 312 & 9.0 & 385 & 24.6 & 697 & 13.9 \\
\hline Other & 11 & 0.3 & 11 & 0.7 & 22 & 0.4 \\
\hline \multicolumn{7}{|l|}{ Degree of Spread } \\
\hline Localised & 197 & 5.7 & 123 & 7.8 & 320 & 6.4 \\
\hline Regional & 232 & 6.7 & 239 & 15.2 & 471 & 9.4 \\
\hline Distant & 38 & 1.1 & 35 & 2.2 & 73 & 1.5 \\
\hline Unknown & 36 & 1.0 & 15 & 1.0 & 51 & 1.0 \\
\hline
\end{tabular}

Tumour location

\begin{tabular}{|c|c|c|c|c|c|}
\hline Upper & 17 & 0.5 & & & 17 \\
\hline Middle & 55 & 1.6 & & & 55 \\
\hline Lower & 331 & 9.6 & & & 331 \\
\hline NOS* $^{*}$ & 99 & 2.9 & & & 99 \\
\hline GOJ* & & & 412 & 26.3 & 412 \\
\hline
\end{tabular}

Charlson comorbidity

\begin{tabular}{lllllll}
0 & 353 & 10.2 & 284 & 18.1 & 637 & 12.7 \\
1,2 & 32 & 0.9 & 22 & 1.4 & 54 & 1.1 \\
$3+$ & 0 & 0.0 & 2 & 0.1 & 2 & 0.0 \\
\hline
\end{tabular}

HOSPITAL CHARACTERISTICS

Surgery type

\begin{tabular}{lllllll} 
Abdothoracic & 79 & 2.3 & 65 & 4.1 & 144 & 2.9 \\
Abdocervical & 64 & 1.9 & 45 & 2.9 & 109 & 2.2 \\
Transhiatal & 360 & 10.4 & 301 & 19.2 & 661 & 13.2 \\
\hline Hospital admission & & & & & & \\
Private co-located & 97 & 2.8 & 75 & 4.8 & 172 & 3.4 \\
Private non co-located & 90 & 2.6 & 87 & 5.5 & 177 & 3.5 \\
Public co-located & 182 & 5.3 & 131 & 8.4 & 313 & 6.2 \\
Public non co-located & 134 & 3.9 & 119 & 7.6 & 253 & 5.0 \\
\hline
\end{tabular}

Table 2 Patient characteristics, location and surgery type of cohort who received resection (Continued)

\begin{tabular}{lllllll}
\hline Hospital area service & & & & & & \\
Area 1 & 99 & 2.9 & 90 & 5.7 & 189 & 3.8 \\
Area 2 & 107 & 3.1 & 85 & 5.4 & 192 & 3.8 \\
Area 3 & 93 & 2.7 & 70 & 4.5 & 163 & 3.2 \\
Area 4 & 126 & 3.6 & 90 & 5.7 & 216 & 4.3 \\
Area 5 & 53 & 1.5 & 51 & 3.3 & 104 & 2.1 \\
Area 6 & 11 & 0.3 & 9 & 0.6 & 20 & 0.4 \\
Area 7 & 14 & 0.4 & 17 & 1.1 & 31 & 0.6 \\
Area 8 & 0 & 0.0 & 0 & 0.0 & 0 & 0.0
\end{tabular}

${ }^{*} \mathrm{NOS}=$ not otherwise specified; $G O \mathrm{~J}=$ gastro-oesophageal junction.

increased complication rates. The loss of statistical significance in the overall cohort may be attributed to the lower complication rate with gastro-oesophageal cancer.

The small number of patients dying within 30-days of surgery did not permit us to perform adjusted analysis for this outcome measure.

\section{Outcomes following surgery in patients with non- metastatic adenocarcinoma}

Rates of hospital length-of-stay $>28$ days $(17.3 \%$ and $19.6 \%), 30$-day mortality (2.4\% and $2.8 \%), 30$-day complication rates $(19.0 \%$ and $19.7 \%)$ and one-year cancer survival $(80.6 \%$ and $79.7 \%)$ were similar for patients with non-metastatic adenocarcinoma oesophageal cancer and the combined cohort respectively.

Factors associated with poorer post-resection outcomes in the sub-cohort were similar to those reported in all patients. However, in this group of patients with non-metastatic adenocarcinoma, we did not observe any significant associations for 30-day post-resection complication in the combined cohort or in oesophageal cancer alone.

\section{Discussion}

There is an increasing use of health administrative data for research and in health policy and planning. We examined rates of oesophagectomy on oesophageal cancer patients and whether the inclusion of patients with gastro-oesophageal cancer affected oesophagectomy rates and post-resection outcomes in NSW. Unsurprisingly, the addition of gastro-oesophageal cancer patients to our cohort increased the oesophagectomy rates by $3.5 \%$. Clearly if hospital funding is based partly on patient admissions and the volume of procedures undertaken, care must be taken to ensure that all potential 'qualifying' patients are included in any performance assessment. Our reported rate of oesophagectomy in patients is comparable to previously reported European and UK studies [30,31] However, the UK rate has been decreasing [38] 
Table 3 Surgery rates and factors associated with receiving surgery by topography

\begin{tabular}{|c|c|c|c|c|}
\hline Variable & $\begin{array}{l}\text { C15 Surgical Rate } \\
\text { (per 100) }\end{array}$ & $\mathrm{aOR}^{\#}(95 \% \mathrm{Cl})$ & $\begin{array}{l}\text { C15\&C160 Surgical } \\
\text { rate (per 100) }\end{array}$ & aOR $(95 \% \mathrm{Cl}){ }^{\#}$ \\
\hline \multicolumn{5}{|l|}{ Age at diagnosis (years) } \\
\hline$<60$ & 25.0 & $8.96(5.63-14.3)^{*}$ & 28.4 & $8.12(5.64-11.7)^{*}$ \\
\hline $60-69$ & 23.2 & $9.85(6.25-15.5)^{*}$ & 26.8 & $8.48(5.94-12.1)^{*}$ \\
\hline 70-79 & 11.1 & $3.99(2.52-6.32)^{*}$ & 15.4 & $4.20(2.94-6.00)^{*}$ \\
\hline $80+$ & 3.1 & Referent & 3.9 & Referent \\
\hline \multicolumn{5}{|l|}{ Gender } \\
\hline Female & 10.2 & Referent & 11.3 & Referent \\
\hline Male & 16.8 & $1.04(0.78-1.38)$ & 21.2 & $1.31(1.05-1.64)^{*}$ \\
\hline \multicolumn{5}{|l|}{ Country of Birth } \\
\hline Australia & 13.8 & Referent & 17.9 & Referent \\
\hline Other & 16.5 & $0.90(0.70-1.16)$ & 19.1 & $0.76(0.62-0.92)^{*}$ \\
\hline \multicolumn{5}{|l|}{ Histology group } \\
\hline SCC & 11.4 & $0.40(0.31-0.52)^{*}$ & 11.7 & $0.41(0.32-0.51)^{*}$ \\
\hline Adenocarcinomas & 20.1 & Referent & 23.6 & Referent \\
\hline Other & 3.8 & $0.28(0.17-0.48)^{*}$ & 5.9 & $0.36(0.24-0.53)^{*}$ \\
\hline \multicolumn{5}{|l|}{ Degree of Spread } \\
\hline Localised & 15.3 & $6.06(4.06-9.06)^{*}$ & 18.6 & $5.50(4.08-7.41)^{*}$ \\
\hline Regional & 33.5 & $12.34(8.22-18.52)^{*}$ & 38.1 & $10.29(7.67-13.79)^{*}$ \\
\hline Distant & 6.1 & Referent & 6.4 & Referent \\
\hline Unknown & 5.0 & $2.38(1.42-3.98)^{*}$ & 5.5 & $1.71(1.14-2.56)^{*}$ \\
\hline \multicolumn{5}{|l|}{ Tumour location } \\
\hline Upper & 5.8 & $0.22(0.12-0.39)$ & 5.8 & $0.22(0.13-0.40)$ \\
\hline Mid & 10.9 & $0.51(0.34-0.76)$ & 10.9 & $0.52(0.35-0.77)$ \\
\hline Distal & 21.5 & Referent & 21.526 .4 & Referent 1.10 (0.88-1.36) \\
\hline \multicolumn{5}{|l|}{ Charlson comorbidity } \\
\hline 0 & 11.4 & Referent & 14.1 & Referent \\
\hline 1,2 & 9.7 & $0.52(0.32-0.83)^{*}$ & 11.9 & $0.56(0.39-0.80)^{*}$ \\
\hline $3+$ & 0 & - & 5.0 & $0.25(0.06-1.14)$ \\
\hline \multicolumn{5}{|l|}{ Hospital admission } \\
\hline Private co-located & 43.1 & Referent & 46.5 & Referent \\
\hline Private non co-located & 4.4 & $0.06(0.04-0.10)^{*}$ & 6.2 & $0.07(0.05-0.10)^{*}$ \\
\hline Public co-located & 34.0 & $0.78(0.53-1.16)$ & 37.8 & $0.80(0.59-1.08)$ \\
\hline Public non co-located & 20.6 & $0.26(0.16-0.42)^{*}$ & 26.5 & $0.26(0.18-0.38)^{*}$ \\
\hline \multicolumn{5}{|l|}{ Hospital area service } \\
\hline Area 1 & 18.1 & $2.30(1.38-3.84)^{*}$ & 23.6 & $2.99(2.03-4.39)^{*}$ \\
\hline Area 2 & 16.2 & $0.73(0.48-1.09)$ & 19.4 & $0.80(0.59-1.09)$ \\
\hline Area 3 & 25.8 & Referent & 28.6 & Referent \\
\hline Area 4 & 19.6 & $1.29(0.86-1.93)$ & 23.8 & $1.58(1.16-2.17)^{*}$ \\
\hline Area 5 & 12.2 & $1.71(0.99-2.97)$ & 16.5 & $1.92(1.27-2.90)^{*}$ \\
\hline Area 6 & 3.5 & $0.82(0.38-1.77)$ & 4.6 & $0.88(0.49-1.56)$ \\
\hline Area 7 & 5.1 & $0.71(0.34-1.47)$ & 8.2 & $0.98(0.59-1.65)$ \\
\hline Area 8 & 0.0 & - & 0.0 & - \\
\hline
\end{tabular}

\footnotetext{
\# Adjusted for age-group, gender, histology, country of birth, histology, degree-of-spread, tumour location, hospital admitted, area of service, Charlson comorbidity index.
} * Significant at $\mathrm{P}<0.05$ (two-tailed) level. 
Table 4 Patient and hospital factors associated with outcomes following surgical resection

\begin{tabular}{|c|c|c|c|c|}
\hline \multirow[b]{2}{*}{ Outcome } & \multicolumn{2}{|l|}{ C15 } & \multicolumn{2}{|l|}{ C15 \& C16.0 } \\
\hline & $\mathrm{N}$ (Rate per 100) & aOR $(95 \% C l)^{\#}$ & $\mathrm{~N}$ (Rate per 100) & aOR $(95 \% \mathrm{Cl}){ }^{\#}$ \\
\hline (i) Length-of-stay $>28$ days & $105(20.9)$ & & $186(19.7)$ & \\
\hline$<60$ years & $23(14.0)$ & $0.20(0.08-0.53)^{*}$ & $43(14.3)$ & $0.24(0.12-0.51)^{*}$ \\
\hline 60-64 years & $43(22.1)$ & $0.41(0.17-1.00)$ & $67(20.2)$ & $0.43(0.22-0.87)^{*}$ \\
\hline 70-74 years & $28(24.1)$ & $0.44(0.17-1.13)$ & $60(25.4)$ & $0.57(0.28-1.17)$ \\
\hline $80+$ years & $11(39.3)$ & Referent & $16(34.8)$ & Referent \\
\hline Australian born & $75(21.7)$ & Referent & $133(20.9)$ & Referent \\
\hline Overseas born & $30(19.0)$ & $0.62(0.38-1.05)$ & $53(19.0)$ & $0.67(0.46-0.99)^{*}$ \\
\hline Private co-located & $14(14.4)$ & Referent & $22(12.8)$ & Referent \\
\hline Private non co-located & $11(12.2)$ & $1.06(0.43-2.63)$ & $23(13.0)$ & $1.92(0.90-4.13)$ \\
\hline Public co-located & $43(23.6)$ & $1.61(0.74-3.49)$ & $71(22.7)$ & $2.10(1.17-3.78)^{*}$ \\
\hline Public non co-located & $37(27.6)$ & $3.16(0.98-10.8)$ & $70(27.7)$ & $7.89(3.16-19.5)^{*}$ \\
\hline Area 1 & $29(29.3)$ & $0.91(0.26-3.18)$ & $52(27.5)$ & $0.44(0.17-1.10)$ \\
\hline Area 2 & $22(20.6)$ & $0.91(0.43-1.96)$ & $43(22.4)$ & $1.24(0.70-2.19)$ \\
\hline Area 3 & $22(23.7)$ & Referent & $37(22.7)$ & Referent \\
\hline Area 4 & $21(16.7)$ & $0.74(0.33-1.66)$ & $33(15.3)$ & $0.64(0.34-1.20)$ \\
\hline Area 5 & $8(15.1)$ & $0.30(0.07-1.26)$ & $14(13.5)$ & $0.15(0.05-0.43)^{*}$ \\
\hline Area 6 & $2(18.2)$ & $0.82(0.12-5.59)$ & $3(15.0)$ & $0.30(0.07-1.39)$ \\
\hline Area 7 & $1(7.1)$ & $0.18(0.02-1.92)$ & $4(12.9)$ & $0.22(0.06-0.86)^{*}$ \\
\hline (ii) 30 day complication & $108(21.5)$ & & $168(17.0)$ & \\
\hline$<60$ years & $35(21.3)$ & $0.40(0.17-0.98)^{*}$ & $53(17.6)$ & $0.53(0.26-1.10)$ \\
\hline $60-64$ years & $36(18.5)$ & $0.33(0.14-0.80)^{*}$ & $59(17.8)$ & $0.55(0.27-1.13)$ \\
\hline 70-74 years & $25(21.6)$ & $0.39(0.16-0.99)^{*}$ & $52(22.0)$ & $0.70(0.34-1.46)$ \\
\hline $80+$ years & $12(42.9)$ & Referent & $14(30.4)$ & Referent \\
\hline Australian born & $71(20.6)$ & Referent & $129(20.3)$ & Referent \\
\hline Overseas born & $37(23.4)$ & $1.28(0.79-2.08)$ & 49 (17.6) & $0.87(0.92-1.06)$ \\
\hline Private co-located & $16(16.5)$ & Referent & $28(16.3)$ & Referent \\
\hline Private non co-located & $22(24.4)$ & $1.13(0.43-2.98)$ & $32(18.1)$ & $1.07(0.51-2.23)$ \\
\hline Public co-located & $40(22.0)$ & $1.82(0.87-3.78)$ & $61(19.5)$ & $1.37(0.79-2.40)$ \\
\hline Public non co-located & $30(22.4)$ & $0.74(0.23-2.37)$ & $57(22.5)$ & $1.47(0.62-3.49)$ \\
\hline Area 1 & $25(25.3)$ & $3.88(1.12-13.5)^{*}$ & $40(21.2)$ & $1.36(0.54-3.47)$ \\
\hline Area 2 & $21(19.6)$ & $1.91(0.85-4.28)$ & $37(19.3)$ & $1.40(0.76-2.57)$ \\
\hline Area 3 & $15(16.1)$ & Referent & $28(17.2)$ & Referent \\
\hline Area 4 & $29(23.0)$ & $2.24(0.99-5.09)$ & $40(18.5)$ & $1.29(0.68-2.42)$ \\
\hline Area 5 & $13(24.5)$ & $4.01(1.04-15.4)^{*}$ & $23(22.1)$ & $1.34(0.49-3.65)$ \\
\hline Area 6 & $9(18.2)$ & $2.86(0.43-19.1)$ & $2(10.0)$ & $0.74(0.14-3.97)$ \\
\hline \multirow[t]{2}{*}{ Area 7} & $11(21.4)$ & $3.87(0.68-22.1)$ & $8(25.8)$ & $2.14(0.66-6.91)$ \\
\hline & Mortality N (rate per 100) & aHR $(95 \% \mathrm{Cl})$ & Mortality N (rate per 100) & aHR $(95 \% \mathrm{Cl})$ \\
\hline (iv) One year survival & $123(24.5)$ & & $218(23.1)$ & \\
\hline$<60$ years & $36(22.0)$ & $0.20(0.10-0.41)^{*}$ & $64(21.3)$ & $0.41(0.23-0.74)^{*}$ \\
\hline 60-64 years & 38 (30.9) & $0.19(0.09-0.38)^{*}$ & 65 (19.6) & $0.36(0.20-0.65)^{*}$ \\
\hline 70-74 years & $32(27.6)$ & $0.22(0.10-0.47)^{*}$ & $68(28.8)$ & $0.44(0.24-0.81)^{*}$ \\
\hline $80+$ years & $17(60.7)$ & Referent & $21(45.6)$ & Referent \\
\hline Australian born & $90(26.1)$ & Referent & $155(24.4)$ & Referent \\
\hline
\end{tabular}


Table 4 Patient and hospital factors associated with outcomes following surgical resection (Continued)

\begin{tabular}{|c|c|c|c|c|}
\hline Overseas born & $33(20.9)$ & $0.64(0.39-1.06)$ & $63(22.6)$ & $0.74(0.51-1.08)$ \\
\hline Private co-located & $10(10.3)$ & Referent & 20 (11.6) & Referent \\
\hline Private non co-located & $22(24.4)$ & $1.58(0.57-4.42)$ & $40(22.6)$ & $2.20(1.03-4.70)^{*}$ \\
\hline Public co-located & $48(26.4)$ & $1.25(0.55-2.83)$ & $80(25.6)$ & $1.80(0.97-3.32)$ \\
\hline Public non co-located & $43(32.1)$ & $1.34(0.45-4.02)$ & $78(30.8)$ & $1.66(0.71-3.89)$ \\
\hline Area 1 & $34(34.3)$ & $1.06(0.36-3.16)$ & $61(32.3)$ & $1.28(0.54-3.03)$ \\
\hline Area 2 & $23(21.5)$ & $0.93(0.42-2.04)$ & $41(21.4)$ & $1.08(0.60-1.96)$ \\
\hline Area 3 & $25(26.9)$ & Referent & $38(23.3)$ & Referent \\
\hline Area 4 & $19(15.1)$ & $0.73(0.32-1.68)$ & $38(17.6)$ & $0.93(0.49-1.76)$ \\
\hline Area 5 & $15(28.3)$ & $0.85(0.26-2.82)$ & $30(28.9)$ & $1.67(0.67-4.11)$ \\
\hline Area 6 & $2(18.2)$ & $1.52(0.27-8.52)$ & $5(25.0)$ & $1.92(0.59-6.22)$ \\
\hline Area 7 & $5(35.7)$ & $1.45(0.36-5.87)$ & $5(16.1)$ & $0.76(0.22-2.66)$ \\
\hline
\end{tabular}

\# Adjusted for age-group, gender, histology, country of birth, degree-of-spread, tumour location, hospital admitted, area of service, Charlson comorbidity index, surgery type.

* Significant at the $P<0.05$ (two-tailed) level.

and may be due to the highly specialised pre- and perioperative assessment and management required for conducting an oesophagectomy [39,40], and the support for centralization of oesophagectomies being performed in dedicated multidisciplinary centres [41,42].

Due to increased precision, the inclusion of gastrooesophageal cancer resulted in an increase in the number of predictor variables associated with rates of resection and post-resection outcomes that were not seen with oesophageal cancer alone (with the exception of 30-day complication rates). For our overall cohort, factors associated with receiving an oesophagectomy included patient characteristics (age, gender, birthplace) cancer characteristics (histology, disease spread) and hospital related factors (location and funding sector); similar to previously published work [31]. 30-day mortality in this study was low, in-line with previous studies and international benchmarks $[9,38,43]$. We found that patients undergoing surgical resection in public hospitals were more likely to have a length of stay $>28$ days. This may reflect the epidemiology of oesophageal and gastro-oesophageal cancer and that more complex cases are treated in public hospitals (teaching hospitals linked to academic centres in Australia). Thus, the addition of gastro-oesophageal cancer did impact on factors associated with receiving an oesophagectomy and on some post-surgery outcomes.

One-year cancer survival was worse in those who underwent surgery in private hospitals that were not colocated with public hospitals, potentially indicating the benefits of surgery being conducted in higher volume or teaching hospitals $[7,9,18,21,30,41,44,45]$.

Our current study demonstrated a reduced 30-day complication rate in gastro-oesophageal cancer compared with oesophageal cancer, hence statistical associations tended towards the null in the combined cohort. This may have been due to the slightly higher rate of transhiatal oesophagectomies undertaken for adenocarcinoma, compared with that in oesophageal cancer and hence lower peri-operative morbidity [46], however the only factors which were significantly associated with higher complication rate in patients with oesophageal cancer were older age and resection being conducted in some area health services.

Although we attempted to account for comorbidity with the use of the CCI, the proportion of patients with comorbidity preceding cancer diagnosis was low and as aforementioned, due to how CCI is calculated, comorbidity was most likely under-ascertained. The underreporting of comorbidities in administrative data may be due to incomplete data transposition from medical records in individual hospitals to administrative databases [47-49]. Further, non-surgical treatments such as chemotherapy and radiotherapy are funded by the PBS and MBS respectively and this data is not linked routinely to hospital morbidity data thus it was not available for this population linkage study. Further, the only measure of disease severity available was degree-of-spread at diagnosis; there were no indicators of performance status available.

\section{Conclusion}

Oesophagectomy in NSW is performed with good outcomes. Our own experience highlights the need for identifying the appropriate patient populations under study and their unique contributions to the overall cohort, given the change in surgical resection rate and longer length-of-stay post-resection with the inclusion of gastrooesophageal cancer. The measurement of hospital performance is a powerful tool to stimulate quality improvement, transparency and accountability and health 
administrative data sets have become pivotal to this activity.

\section{Competing interests}

The authors declare that they have no competing interests.

\section{Authors' contributions}

ES helped to design the study, conducted the statistical analysis and drafted the manuscript. SAP and RW designed the study and helped to draft the manuscript. All authors read and approved the final manuscript.

\section{Acknowledgements}

The authors wish to acknowledge NSW Health for use of the data and the CHeReL for undertaking the data linkage. This study was partially funded by the Cancer Services Cancer Institute NSW (block funding agreement with South East Sydney \& Illawarra Area Health Service). The funders had no role in the study design, data collection, data analysis, data interpretation, or writing of the manuscript. The views expressed are not necessarily those of the funders.

\section{Author details}

'Lowy Cancer Research Centre, Prince of Wales Clinical School, University of New South Wales, Sydney, NSW, Australia. ${ }^{2}$ Faculty of Pharmacy, University of Sydney, Sydney, NSW, Australia. ${ }^{3}$ Kolling Institute of Medical Research,

University of Sydney, St Leonards, NSW 2065, Australia.

Received: 12 June 2012 Accepted: 31 October 2012

Published: 8 November 2012

\section{References}

1. Berwick DM, Leape LL: Reducing errors in medicine. BMJ 1999, 319:136-137.

2. Leathley CM, Gilbert R, Kennedy PJ, Hughes CF: Measuring hospital performance - 2008 forum summary. Med J Aust 2010, 193:S95-S96.

3. Clinical Excellence Commission (CEC) and NSW Department of Health: Clinical Incident Management in the NSW Public Health System 2009. Sydney: NSW Department of Health; 2010.

4. Department of Health and Human Services Agency for Healthcare Research and Quality: Guide to Inpatient Quality Indicators: Quality of care in hospitals volume, mortality, and utilization. Rockville: Department of Health and Human Services; 2007

5. Ra J, Paulson EC, Kucharczuk J, Armstrong K, Wirtalla C, Rapaport-Kelz R, Kaiser LR, Spitz FR: Postoperative mortality after esophagectomy for cancer: Development of a preoperative risk prediction model. Ann Surg Oncol 2008, 15(6):1577-1584.

6. Reavis KM, Smith BR, Hinojosa MW, Nguyen NT: Outcomes of Esophagectomy at Academic Centers: An Association between Volume and Outcome. Am Surg 2008, 74(10):939-943.

7. Verhoef $C$, van de Weyer R, Schaapveld M, Bastiaannet E, Plukker JTM: Better survival in patients with oesophageal cancer after surgical treatment in University hospitals: A plea for performance by surgical oncologists. Ann Surg Oncol 2007, 14:1678-1687.

8. Spilsbury K, Semmens JB, Saunders CM, Holman CDJ: Long-term survival outcomes following breast cancer surgery in Western Australia. ANZ Surg 2005, 75(8):U625-U624.

9. Stavrou EP, Smith GS, Baker DF: Surgical outcomes associated with oesophagectomy in New South Wales; an investigation of hospital volume. J Gastrointest Surg 2010, 14:951-957.

10. Ben-Tovim DI, Pointer SC, Woodman R, Hakendorf PH, Harrison JE: Routine use of administrative data for safety and quality purposes - hospital mortality. Med J Aust 2010, 193:S100-S103.

11. Chowdhury MM, Dagash H, Pierro A: A systematic review of the impact of volume of surgery and specialization on patient outcome. Br J Surg 2007, 94(2):145-161.

12. Finlayson EVA, Goodney PP, Birkmeyer JD: Hospital volume and operative mortality in cancer surgery - A national study. Archives Surg 2003, 138(7):721-725.

13. Holman CDJ, Wisniewski ZS, Semmens JB, Rouse IL, Bass AJ: Mortality and prostate cancer risk in 19598 men after surgery for benign prostatic hyperplasia. BJU Int 1999, 84(1):37-42.

14. Karanicolas PJ, Dubois L, Colquhoun PHD, Swallow CJ, Walter SD, Guyatt $\mathrm{GH}$ : The more the better? The impact of surgeon and hospital volume on in-hospital mortality following colorectal resection. Ann Surg 2009, 249(6):954-959.

15. Reavis KM, Hinojosa MW, Smith BR, Wooldridge JB, Krishnan S, Nguyen NT: Hospital volume is not a predictor of outcomes after gastrectomy for neoplasm. Am Surg 2009, 75(10):932-936.

16. Shahian DM, O'Brien SM, Normand SLT, Peterson ED, Edwards FH: Association of hospital coronary artery bypass volume with processes of care, mortality, morbidity, and the Society of Thoracic Surgeons composite quality score. J Thoracic Cardiovasc Surg 2010, 139(2):273-282.

17. Smith FJ, Holman CDJ, Moorin RE, Fletcher DR: Incidence of bariatric surgery and postoperative outcomes: a population-based analysis in Western Australia. Med J Aust 2008, 189(4):198-202.

18. Bachmann MO, Alderson N, Edwards D, Wotton S, Bedford C, Peters TJ, et al: Cohort study in South and West England of the influence of specialization on the management and outcome of patients with oesophageal and gastric cancers. Br J Surg 2002, 89:914-922.

19. Pye JK, Crumplin MKH, Charles J, Kerwat R, Foster ME, Biffin A: One-year survey of carcinoma of the oesophagus and stomach in Wales. Br J Surg 2001, 88:278-285.

20. Swisher SG, Deford L, Merriman KW, Walsh GL, Smythe R, Vaporicyan A, al E: Effect of operative volume on morbidity, mortality and hospital use after esophagectomy for cancer. J Thorac Cardivasc Surg 2000, 119:1126-1132.

21. van Lanschot JJB, Hulscher JB, Buskens CJ, Tilanus HW, ten Kate FJW Obertop H: Hospital volume and hospital mortality for esophagectomy. Cancer 2001, 91:1574-1578.

22. Wouters M, Krijnen P, Le Cessie S, Gooiker GA, Guicherit OR, Marinelli A, Kievit J, Tollenaar R: Volume- or Outcome-Based Referral to Improve Quality of Care for Esophageal Cancer Surgery in The Netherlands. J Surg Oncol 2009, 99(8):481-487.

23. Ajani J, Barthel JS, Bekaii-Saab T, Bentrem D, D'Amico TA, Das P, et al: Gastric Cancer, NCCN Practice Guidelines in Oncology v22010. Fort Washington: National Comprehensive Cancer Network; 2010.

24. Ajani J, Barthel JS, Bekaii-Saab T, Bentrem DJ, D'Amico TA, Das P, et al: Esophageal Cancer, NCCN Clinical Practice Guidelines in Oncology v12010. Fort Washington: National Comprehensive Cancer Network; 2010.

25. Stavrou EP, McElroy HJ, Baker DF, Bishop JF: Adenocarcinoma of the oesophagus: incidence and survival rates in New South Wales, 1972-2005. Med J Aust 2009, 191:310-314.

26. Semmens JB, Lawrence-Brown MMD, Fletcher DR, Rouse IL, Holman CDJ: The quality of surgical care project: A model to evaluate surgical outcomes in Western Australia using population-based record linkage. ANZ J Surg 1998, 68(6):397-403.

27. Semmens JB, Aitken RJ, Sanfilippo FM, Mukhtar SA, Haynes NS, Mountain JA The Western Australian audit of surgical mortality: advancing surgical accountability. Med J Aust 2005, 183:504-508.

28. Board N, Watson DE: Using what we gather - harnessing information for improved care. Med J Aust 2010, 193:S93-S94.

29. Semmens JB, Mountain JA, Sanfilippo FM, Barraclough JY, McKenzie A, Mukhtar SA, Haynes NS, Aitken RJ: Providers and consumers support the Western Australian Audit of surgical mortality. ANZ J Surg 2006, 76:442-447.

30. Gillison EW, Powell J, McConkey CC, Spychal RT: Surgical workload and outcome after resection for carcinoma of the oesophagus and cardia. Br J Surg 2002, 89:344-348.

31. Verhorf C, van de Weyer R, Schaapveld M, Bastiaannet E, Plukker JTM: Better survival in patients with oesophageal cancer after surgical treatment in University hospitals: A plea for performance by surgical oncologists. Ann Surg Oncol 2007, 14:1678-1687.

32. International Agency for Research on Cancer: Manual for Cancer Registry Personnel: IARC Technical Report No 10. Lyon: IARC 1995, 81:1-4.

33. Barraclough H, Morrell S, Arcorace M, McElroy H, Baker DF: Degree-ofspread artefact in the New South Wales Central Cancer Registry. Aust NZ J Public Health 2008, 32:414-416.

34. National Centre for Classification in Health: ICD-10-AM/ACHI/ACS. Sixth edition. Sydney: University of Sydney; 2008

35. Charlson ME, Pompei P, Ales KL, McKenzie CR: A new method of classifying prognostic comorbidity in longitudinal studies: development and validation. J Chronic Dis 1987, 40:373-383

36. Deyo RA, Cherkin DC, Ciol MA: Adapting a clinical co-morbidity index for use with ICD-9-CM administrative databases. J Clin Epidemiol 1992, 45:613-619. 
37. Quan $\mathrm{H}$, Sundararajan $\mathrm{V}$, Halfon $\mathrm{P}$, Fong A, Burnand B, Luthi JC, et al: Coding algorithms for defining comorbidities in ICD-9-CM and ICD-10 administrative data. Med Care 2005, 43:1130-1139.

38. Allum WH, Blazeby JM, Griffin SM, Cunningham D, Jankowski JA, Wong R: On behalf of the Association of Upper Gastrointestinal Surgeons of Great Britain and Ireland, the British Society of Gastroenterology and the British Association of Surgical Oncology: Guidelines for the management of oesophageal and gastric cancer. Gut 2011, 60:1449-1472.

39. Morita M, Nakanoko T, Fujinaka Y, Kubo N, Yamashita Y, Yoshinaga K, et al: In-hospital mortality after a surgical resection for esophageal cancer: Analyses of the associated factors and historical changes. Ann Surg Oncol 2011, 18:1757-1765.

40. Fujita H, Ozawa S, Kuwano H, Ueda Y, Hattori S, Yanagawa T: Japanese Assoc Thoracic S: Esophagectomy for cancer: clinical concerns support centralizing operations within the larger hospitals. Dis Esophagus 2010 23(2):145-152.

41. Rouvelas I, Lagergren J: The impact of volume on outcomes after oeseophageal cancer surgery. ANZ J Surg 2010, 80:634-641.

42. Anderson O, Ni Z, Moller H, Coupland VH, Davies EA, Allum WH, Hanna GB; Hospital volume and survival in oesophagectomy and gastrectomy for cancer. Eur J Cancer 2011, 47:2408-2414.

43. Royal College of Surgeons of England: National Oesophago-Gastric Cancer Audit. Third Annual Report. London: The NHS Information Centre; 2010:1-69.

44. Wouters MW, Wijnhoven BP, Karim-Kos HE, Blaauwgeers HG, Stassen LP, Steup W-H, et al: High-volume versus low-volume for esophageal resections for cancer: The essential role of case-mix adjustments based on clinical data. Ann Surg Oncol 2008, 15:80-87.

45. Fujita H, Kuwano H, Ueda Y, Hattori S, Yanagawa T: Esophagectomy for cancer: clinical concerns support centralizing operations within the larger hospitals. Dis Esophagus 2010, 23:145-152.

46. Hulscher JB, van Sandick JW, de Boer AG, Wijnhoven BP, Tijssen JG, Fockens $P$, et al: Extended transthoracic resection compared with limited transhiatal resection for adenocarcinoma of the esophagus. N Engl I Med 2002, 347:1662-1669.

47. Preen DB, Holman CDJ, Lawrence DM, Baynham NJ, Semmens JB: Hospital chart review provided more accurate comorbidity information than data from a general practitioner survey or an administrative database. J Clin Epidemiol 2004, 57:1295-1304.

48. Powell H, Lim LL-Y, Heller RF: Accuracy of administrative data to assess comorbidity in patients with heart disease: an Australian perspective. J Clin Epidemiol 2001, 54:687-693.

49. Hawker GA, Coyte PC, Wright JG, Paul JE, Bombardier C: Accuracy of administrative data for assessing outcomes after knee replacement surgery. J Clin Epidemiol 1997, 50:265-273.

\section{doi:10.1186/1472-6963-12-384}

Cite this article as: Stavrou et al:: Oesophagectomy rates and postresection outcomes in patients with cancer of the oesophagus and gastro-oesophageal junction: a population-based study using linked health administrative linked data. BMC Health Services Research 2012 12:384.

\section{Submit your next manuscript to BioMed Central and take full advantage of:}

- Convenient online submission

- Thorough peer review

- No space constraints or color figure charges

- Immediate publication on acceptance

- Inclusion in PubMed, CAS, Scopus and Google Scholar

- Research which is freely available for redistribution 